\title{
Implementation of a new telephone triage system in ophthalmology emergency department during COVID-19 pandemic: clinical effectiveness, safety and patient satisfaction
}

\author{
Yunzi Chen $\mathbb{1}^{1} \cdot$ Rehab Ismail $^{1} \cdot$ Muhammad R. Cheema $\mathbb{1}^{1} \cdot$ Darren S. J. Ting $\mathbb{(}^{2,3} \cdot$ Ibrahim Masri $^{1}$
}

Received: 17 January 2021 / Revised: 28 February 2021 / Accepted: 31 March 2021 / Published online: 25 May 2021

(c) The Author(s), under exclusive licence to The Royal College of Ophthalmologists 2021

\section{To the Editor:}

Since the WHO declaration of COVID-19 pandemic in March 2020, various countries have implemented regional and/or national lockdown with stringent rules on social distancing to minimise the risk of transmission. This has significantly affected the service delivery across all medical fields, including ophthalmology [1,2]. In addition, studies have shown that patient would delay seeking ocular treatment due to fear of contracting COVID-19 infection [3, 4].

In response to the COVID-19 pandemic, we had reformed our eye emergency service in 2020 by introducing a new telephone triage system (TSS) to cope with the reduced health service capacity and protect patients and front-line staff, aligned with the national and Royal College guidance [5]. All patients were triaged and assessed via TTS before being accepted for face-to-face consultation if deemed necessary. In this study, we aimed to report the effectiveness, safety, and acceptability of this new TSS.

All telephone triage encounters and attendances to the eye emergency department (EED) at Sunderland Eye Infirmary, UK, during April-May 2020 were included. Relevant data, including the number of attendances, waiting times, presenting diagnoses and patient/staff satisfaction

Supplementary information The online version contains supplementary material available at https://doi.org/10.1038/s41433021-01528-8.

Yunzi Chen

chen_yunzi@hotmail.com

1 Sunderland Eye Infirmary, Sunderland, UK

2 Academic Ophthalmology, Division of Clinical Neuroscience, University of Nottingham, Nottingham, UK

3 Department of Ophthalmology, Queen's Medical Centre, Nottingham, UK (assessed via questionnaires-Supplementary Material), were analysed. The safety of TSS was examined via analysis of repeat callers (patients who accessed the TSS more than once for the same complaint) and their final diagnosis at EED. Ethical approval was not required as this retrospective study was considered a clinical service evaluation study.

Of all 2682 phone calls ( $n=2293$ patients), $52 \%$ were managed successfully via TSS and $48 \%$ subsequently reviewed at EED. Compared to 2019, there was a significant $65 \%$ reduction in overall EED attendances $(p<0.001$; Table 1). Mean arrival-to-treatment time significantly reduced by $43 \mathrm{~min}$. There was a significant increase in ocular trauma and reduction in oculoplastic and conjunctival diagnosis $(p<0.001)$.

There were $169(7 \%)$ repeat callers (Table 2), with a higher proportion in older age groups. Of those, 90 (53\%) patients attended EED eventually. Following the review, only $7(0.3 \%)$ triage decisions out of 2682 calls were considered inappropriate and classed as missed diagnosis. These included retinal detachment $(n=2)$, contact lensrelated infectious keratitis $(n=1)$, wet age-related macular degeneration $(n=1)$, non-ischaemic anterior ischaemic optic neuropathy $(n=1)$, macular hole $(n=1)$ and marginal keratitis $(n=1)$. In all cases, the triage decisions were made by the consultants. Potential harm to vision was considered avoidable in four cases if the patient was reviewed earlier at EED.

A total of 69 (17\%) patients responded to the survey. The majority (96\%) of patients described their experience of using TSS as good-to-outstanding, $88 \%$ indicated that they would use the service again, $91 \%$ recommended the service, and $93 \%$ recommended the continuation of the TSS. Of 36 (64\%) responded staff, $94 \%$ described a positive experience with the TSS and $100 \%$ recommended the future use of TSS.

The COVID-19 pandemic has posed an unprecedented challenge to ophthalmic service delivery due to cancellation 
Table 1 Summary of the patient attendances to eye emergency department in April-May 2019 and April-May 2020.

\begin{tabular}{|c|c|c|c|}
\hline Parameters & $\begin{array}{l}2019 \\
\text { Total } \\
N=4566 \\
N(\%)\end{array}$ & $\begin{array}{l}2020 \\
\text { Total } \\
N=1342 \\
N(\%)\end{array}$ & $P$ value \\
\hline Gender & & & $\leq 0.001$ \\
\hline Female & $2312(50.6)$ & $597(44.5)$ & \\
\hline Male & $2254(49.4)$ & $745(55.5)$ & \\
\hline Age, years & & & $\leq 0.001$ \\
\hline $0-29$ & $965(21.1)$ & $241(17.9)$ & \\
\hline $30-49$ & $1159(25.4)$ & $385(28.7)$ & \\
\hline $50-60$ & $1465(32.1)$ & $497(37.0)$ & \\
\hline$>70$ & 977 (21.4) & $219(16.3)$ & \\
\hline Types of attendance & & & $\leq 0.001$ \\
\hline New attendance & $4104(90.0)$ & $1250(93.1)$ & \\
\hline Planned follow-up & $236(5.2)$ & $61(4.6)$ & \\
\hline Unplanned follow-up & $226(4.9)$ & $31(2.3)$ & \\
\hline Mean time to assessment, mins ${ }^{b}$ & $11.9 \pm 1.0$ & $6.9 \pm 0.9$ & $\underline{0.033}$ \\
\hline Mean time to treatment, $\operatorname{mins}^{\mathrm{c}}$ & $76.5 \pm 9.3$ & $33.8 \pm 6.4$ & $\underline{0.042}$ \\
\hline Out of hours attendances ${ }^{\mathrm{d}}$ & $916(20.1)$ & $217(16.2)$ & $\leq 0.001$ \\
\hline EED diagnosis & & & $\leq 0.001$ \\
\hline Trauma & $781(17.1)$ & $368(27.4)$ & \\
\hline Corneal & $742(16)$ & $213(16)$ & \\
\hline Retinal & $537(12)$ & $157(12)$ & \\
\hline Conjunctival & $783(17.1)$ & $127(9.5)$ & \\
\hline Oculoplastic & $682(14.9)$ & $149(11.1)$ & \\
\hline Others & $1041(22.8)$ & $328(75.6)$ & \\
\hline Disposal description & & & $\leq 0.001$ \\
\hline Discharge without follow-up & $3291(72.1)$ & $1009(75.2)$ & \\
\hline Referred to clinic & $579(12.7)$ & $178(13.3)$ & \\
\hline Review in EED & $280(6.1)$ & $79(5.9)$ & \\
\hline Referred to GP & $115(2.5)$ & $46(3.4)$ & \\
\hline Admitted & $31(0.7)$ & $16(1.2)$ & \\
\hline Others & $270(5.9)$ & $14(1.0)$ & \\
\hline
\end{tabular}

${ }^{\mathrm{a} P}$ values are calculated using Chi-square test (for categorical variables) and unpaired $T$ test (for continuous variables). Continuous values are presented in mean \pm standard deviation. Significant $p$ values are underlined.

${ }^{\mathrm{b}}$ Time from arrival to assessment.

${ }^{\mathrm{c}}$ Time from arrival to treatment.

${ }^{\mathrm{d}}$ Defined as between 17:00 till 8:00 next day.

of the routine services. To the best of our knowledge, this study serves as one of the few UK studies that examined the effectiveness and safety of TSS in a previously open-access EED during COVID-19 pandemic. Our data showed that TSS served as an effective model to triage the patients, with only $\sim 50 \%$ converting to face-to-face consultation, paralleling with the findings in literature [6]. Furthermore, analysis of diagnoses demonstrated that there were fewer minor conditions being seen whilst the proportion of more serious
Table 2 Triage outcome and level of triage assessor at repeat callers' 1 st and 2nd triage encounter.

\begin{tabular}{lcc}
\hline Parameters & $\begin{array}{c}\text { 1st Triage } \\
\text { Total } N=169 \\
N(\%)\end{array}$ & $\begin{array}{c}\text { 2nd Triage } \\
\text { Total } N=169 \\
N(\%)\end{array}$ \\
\hline Triage outcome & & \\
$\quad$ EED assessment & $0(0)$ & $81(48.2)$ \\
Over the counter treatment & $85(50.6)$ & $35(20.8)$ \\
Monitor symptoms & $46(27.4)$ & $23(13.7)$ \\
$\quad$ Referred to other services & $33(19.6)$ & $24(14.2)$ \\
$\quad$ Others & $5(2.9)$ & $6(3.6)$ \\
Grade of triage assessor & & $127(75.1)$ \\
$\quad$ Consultant & $125(74.0)$ & $14(8.3)$ \\
Registrar & $10(5.9)$ & $20(11.8)$ \\
$\quad$ Nurse practitioner & $20(11.8)$ & $8(4.7)$ \\
$\quad$ Not recorded & $14(8.3)$ & \\
\hline
\end{tabular}

or urgent ophthalmic diagnoses were maintained. We also observed a dramatic improvement in waiting time compared to before, which allowed strict social distancing precautions to take place and protect vulnerable patients from infection exposures.

Notably, only $0.3 \%$ triage decisions were considered to be inappropriate. This was similar to a Paris study, which reported $1 \%$ of misdiagnosis of teleconsultation in EED leading to delayed ophthalmic care during COVID-19 lockdown [7]. We observed two missed cases of retinal detachment, highlighting the difficulty in safely triaging patients complaining of flashes and floaters. Furthermore, all missed diagnoses were made by consultants, suggesting that reliance on experience alone may not be sufficient to guarantee safety and a consistent triage protocol will be required. That said, nearly all patients and staff expressed high level of satisfaction with the TSS in view of the perceived benefits of immediate access to advice, reduced waiting time, prioritisation of true emergencies, and low risk of COVID-19.

Considering the persistent COVID-19 pandemic with further waves of infection, healthcare digitalisation using tele-ophthalmology (with potential integration of artificial intelligence) is emerging as a potentially long-term solution to assessing and managing ophthalmic diseases at the frontline service while minimising the risk of COVID-19 [8-10].

Author contributions $\mathrm{YC}$ and IM contributed to the study conception and design. Material preparation, data collection and analysis were performed by YC, RI, MC, DT and IM. The first draft of the paper was written by YC, DT and IM and all authors commented on previous versions of the paper. All authors read and approved the final paper.

\section{Compliance with ethical standards}

Conflict of interest The authors declare no competing interests. 
Publisher's note Springer Nature remains neutral with regard to jurisdictional claims in published maps and institutional affiliations.

\section{References}

1. Ting DSJ, Deshmukh R, Said DG, Dua HS. The impact of COVID-19 pandemic on ophthalmology services: are we ready for the aftermath? Ther Adv Ophthalmol. 2020;12:1-3.

2. Romano MR, Montericcio A, Montalbano C, Raimondi R, Allegrini D, Ricciardelli G, et al. Facing COVID-19 in ophthalmology department. Curr Eye Res. 2020;45:653-8.

3. Ting DSJ, Krause S, Said DG, Dua HS. Psychosocial impact of COVID-19 pandemic lockdown on people living with eye diseases in the UK. Eye. 2020. https://doi.org/10.1038/s41433-02001130-4.

4. Wickham L, Hay G, Hamilton R, Wooding J, Tossounis H, da Cruz L, et al. The impact of COVID policies on acute ophthalmology services-experiences from Moorfields. Eye Hospital NHS Found Trust Eye. 2020;34:1189-92.

5. RCOphth. The Royal College of Ophthalmologists Protecting Patients, Protecting Staff. 2020. https://www.rcophth.ac.uk/wp-
content/uploads/2020/03/Protecting-Patients-Protecting-StaffUPDATED-300320.pdf.

6. Bunn F, Byrne G, Kendall S. Telephone consultation and triage: effects on health care use and patient satisfaction. Cochrane Database Syst Rev. 2004:CD004180. https://doi.org/10.1002/ 14651858.CD004180.pub2.

7. Bourdon H, Jaillant R, Ballino A, El Kaim P, Debillon L, Bodin S, et al. Teleconsultation in primary ophthalmic emergencies during the COVID-19 lockdown in Paris: experience with 500 patients in March and April 2020. J Fr Ophtalmol. 2020;43:577-85.

8. Ting DSJ, Foo VHX, Yang LWY, Sia JT, Ang M, Lin H, et al. Artificial intelligence for anterior segment diseases: emerging applications in ophthalmology. $\mathrm{Br} \mathrm{J}$ Ophthalmol. 2021;105:158-68

9. Ting DSJ, Ang M, Mehta JS, Ting DSW. Artificial intelligenceassisted telemedicine platform for cataract screening and management: A potential model of care for global eye health. Br J Ophthalmol. 2019;103:1537-8.

10. Wu X, Chen J, Yun D, Yuan M, Liu Z, Yan P, et al. Effectiveness of an ophthalmic hospital-based virtual service during the COVID-19 pandemic. Ophthalmology. 2021. https://doi.org/10. 1016/j.ophtha.2020.10.012. 